\title{
A peripheral primitive neuroectodermal tumor in the larynx: A case report and literature review
}

\author{
KEI IJICHI $^{1}$, TOYONORI TSUZUKI ${ }^{2}$, MAKOTO ADACHI $^{3}$ and SHINGO MURAKAMI $^{1}$ \\ ${ }^{1}$ Department of Otolaryngology - Head and Neck Surgery, Nagoya City University Graduate School of Medical Sciences, \\ Nagoya, Aichi 467-8601; ${ }^{2}$ Department of Pathology, Nagoya Daini Red Cross Hospital, Nagoya, Aichi 466-8650; \\ ${ }^{3}$ Department of Oral and Maxillofacial Surgery, Asahi University Murakami Memorial Hospital, Gifu 500-8856, Japan
}

Received December 23, 2014; Accepted August 26, 2015

DOI: $10.3892 / \mathrm{ol} .2015 .4013$

\begin{abstract}
Primitive neuroectodermal tumors (PNETs) are malignant tumors comprised of small round cells of neuroectodermal origin. Current evidence indicates that peripheral PNETs (pPNETs), which arise in the non-central nervous system, possess histological similarity to Ewing's sarcoma. Though the occurrence of pPNETs in the head and neck region is rare, these are aggressive malignant tumors, and long-term survival rates following diagnosis remain poor. The current report presents a case of pPNET and evaluates its significance with regard to previous studies. In the present case, a tumor was located in the larynx of the patient, and was diagnosed as pPNET. Immunohistochemical analysis indicated that tumor cells were positive for cluster of differentiation 99. The patient was treated with surgery, multiagent chemotherapy and radiotherapy. Five years subsequent to treatment, the patient had survived and demonstrated no evidence of disease recurrence. In existing literature concerning pPNET located outside the head and neck region, it is recommended that patients are treated with a combination of resection with a wide surgical margin, multiagent chemotherapy and radiotherapy. The present case report concluded that the combination of surgery, systematic chemotherapy and radiotherapy, offers an improved outcome for pPNET localized to the head and neck region, compared with any of these therapies alone.
\end{abstract}

Correspondence to: Dr Kei Ijichi, Department of Otolaryngology - Head and Neck Surgery, Nagoya City University Graduate School of Medical Sciences, 1 Kawasumi, Mizuho-cho, Mizuho-ku, Nagoya, Aichi 467-8601, Japan

E-mail: ijichi@med.nagoya-cu.ac.jp

Key words: primitive neuroectodermal tumor, peripheral primitive neuroectodermal tumor, Ewing's sarcoma, larynx, head and neck sarcoma, head and neck

\section{Introduction}

Primitive neuroectodermal tumor (PNET) is a small round cell sarcoma that primarily develops in the central nervous system (CNS) and soft tissues of children (1). In particular, peripheral PNET (pPNET) has been hypothesized to originate from the neural crest (2). Previously, PNET was suggested to be a neoplasm of the central nervous system (3). Since then, this concept has been extended to encompass the periphery, and these non-central nervous system tumors are referred to as pPNETs (3). pPNET was initially described by Stout in 1918 (4). In addition, Ewing's sarcoma (ES) of the bone was initially reported by J. Ewing in 1921 (5). Subsequent to this initial description, a diagnosis of extraskeletal ES was reported by Angervall and Enzinger in 1975 (6). In 1979, Askin et al (7) described a 'malignant small cell tumor of the thoracopulmonary region' (Askin tumor). An additional significant discovery occurred in 1984, when a neuroectodermal tumor of the bone was identified by Jaffe et al (8). At the time of diagnosis, these aforementioned tumors were recognized as being pathologically distinct. However, it has since become apparent that these tumors share a number of pathological features $(7,8)$.

ES and pPNET (including Askin tumor) are proposed to arise from the neural crest (9). Currently, there are two opposing opinions that recognize ES and pPNET as separate entities, or alternatively group them within a single category known as the 'ES/pPNET group' (3) or the 'ES family of tumors' (10). Pathologically, when ES and pPNET are recognized as separate entities, these distinctions are based primarily on the more neural differentiation exhibited by pPNET (11).

Typically, when considered from a histopathological perspective, pPNETs possess similarities to a number of neoplasms, including rhabdomyosarcoma and small cell carcinoma (4).

Within the head and neck region, the larynx is a rare primary site for pPNET to arise (12). To the best of our knowledge, only a small number of cases have previously been reported (13-15). In these cases, follow-up periods were short and the details of treatment were not discussed. The current report presents a case of pPNET arising in the larynx, and subsequently reviews the associated literature. 
Table I. Immunohistochemical analysis results.

\begin{tabular}{lc}
\hline Antibody & Result \\
\hline Glycogen & - \\
Vimentin & + \\
S100 protein & + \\
Neuron-specific enolase & + \\
Cluster of differentiation 99 & + \\
Leucocyte common antigen & - \\
Desmin & - \\
a-smooth actin & - \\
Synaptophysin & - \\
Muscle actin & - \\
Chromogranin & - \\
\hline
\end{tabular}

\section{Case report}

A previously healthy 33-year-old female presented with the symptom of hoarseness $\sim 6$ months prior to admission. This symptom was progressive, and the patient was unable to speak during the initial consultation. Following clinical examination using a fiberscope, a slightly red tumor covered with white tissue was identified on the right false vocal cord.

A punch biopsy was performed under fiberscopic view, and the tumor was initially diagnosed as being neuroendocrine. However, this diagnosis was unreliable due to the small size of the biopsy specimen. In addition, the initial report indicated that the tumor possessed low malignant potential. Computed tomography (CT) and magnetic resonance imaging (MRI) studies were unable to detect the tumor due to its small size.

Consequently, microscopic surgery was performed under general anesthesia after admission to Nagoya Daini Red Cross Hospital on June 28, 2001. The base of the tumor mass was identified in the right laryngeal ventricle (Fig. 1), and dissected from the mucosa. The tumor was resected, and subsequently a potassium titanyl phosphate laser beam was applied to the tumor resection and the mucosa around the tumor. A diagnosis of pPNET was reached following pathological examination. Paraffin sections revealed sheets of small round cells, which possessed relatively regular nuclei and sparse cytoplasm. The nuclei demonstrated a salt and pepper chromatin pattern, and the nucleoli were obscured. Abortive Homer-Wright rosettes were rare, and no other specific patterns were identified. A relatively high frequency of mitotic figures was observed, while a Periodic Acid Schiff stain indicated that no glycogen was present (Fig. 2A). Immunohistochemical studies revealed that tumor cells were positive for cluster of differentiation 99 (CD99), neuron-specific enolase (NSE), S100 protein and vimentin, and negative for cytokeratin (AE1/AE3, CAM5.2), epithelial membrane antigen, muscle-specific actin, $\alpha$-smooth muscle actin, desmin, leucocyte common antigen, chromogranin A and synaptophysin (Fig. 2B; Table I). An ultrastructual study revealed that the tumor cytoplasm contained a reduced number of mitochondria. Other organelles were not well developed; no electron dense core granules were detected, and nuclei were round with obscured nucleoli.

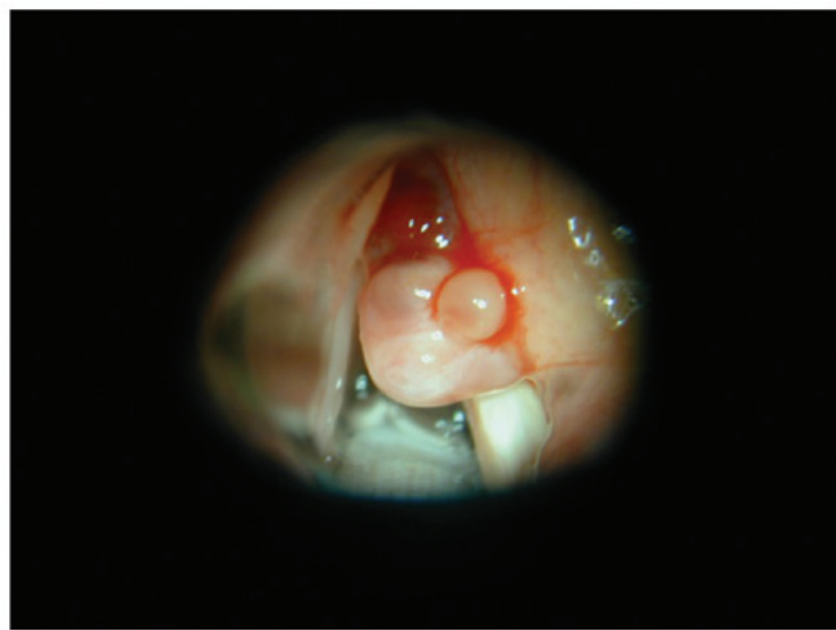

Figure 1. Intraoperative image of the area above the right vocal cord. The mass was based at the right laryngeal ventricle. The tumor was soft and easily hemorrhagic.

A

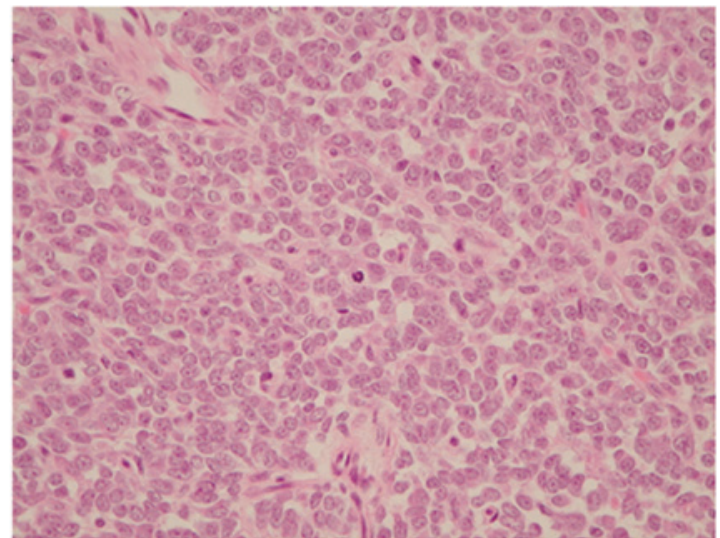

B

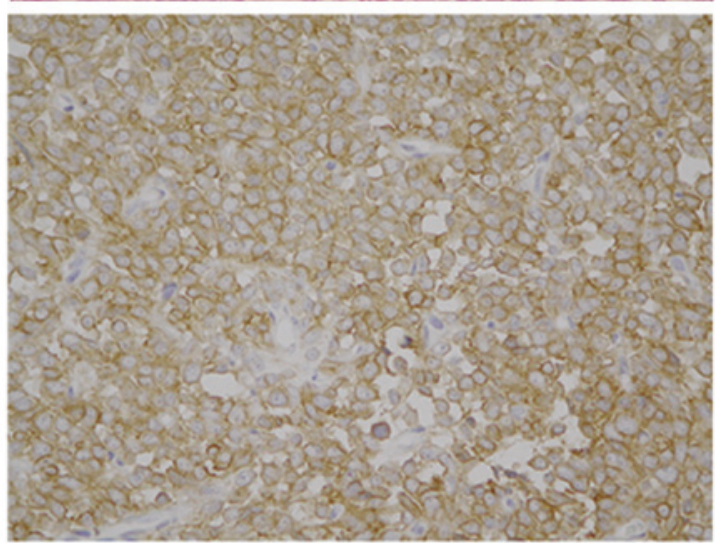

Figure 2. Histopathological and immunohistochemical tumor staining. (A) Hematoxylin and eosin-stained sections of the tumor. The tumor was comprised of small round cells with relatively regular nuclei and sparse cytoplasm. Abortive Homer-Wright rosettes were rarely observed. (B) Immunohistochemistry for cluster of differentiation 99 demonstrated positive membranous staining of tumor cells. Magnification, x200.

Following diagnosis, CT scans of the chest, abdomen and pelvis, an X-ray of all bones, an isotope bone scan, and an MRI scan of the brain and bone marrow, were normal. Hematological tests revealed a slight increase in the levels of lactate dehydrogenase and creatine phosphokinase. 
Table II. Review of reported cases of laryngeal peripheral primitive neuroectodermal tumors and the present case.

\begin{tabular}{lcccccc}
\hline Study & Year & Patient age, years & Patient gender & Treatment & Metastases & Reference \\
\hline Jones et al & 1995 & 9 months & M & S+C & None & $(13)$ \\
Yang et al & 2004 & 74 & M & S+R & None & $(14)$ \\
Lynch et al & 2014 & 45 & F & C+R & None & $(15)$ \\
Present case & & 33 & F & S+C+R & None & \\
\hline
\end{tabular}

C, chemotherapy; R, radiation therapy; S, surgery; M, male; F, female.

Additional surgery with a wide marginal resection, including a total laryngectomy, was recommended; however, the patient declined. The patient was therefore treated with multiagent chemotherapy (ifosfamide, $50 \mu \mathrm{g} / \mathrm{kg}$; vincristine, $2 \mu \mathrm{g} / \mathrm{kg}$; doxorubicin, $500 \mu \mathrm{g} / \mathrm{kg}$ ). The patient also received radiotherapy of a total dose of 60 Gy over 6 weeks.

The patient currently remains alive and healthy. Five years subsequent to treatment, the patient has exhibited no local disease recurrence or evidence of metastases.

\section{Discussion}

The concept of PNET has been the subject of considerable discussion in multiple cases $(8,9)$. Initially, the term PNET was limited to tumors of the CNS. However, non-CNS tumors possessing a similar histological origin have been reported, and are known as pPNETs (3).

pPNET, with a primary site in the head and neck region, is rarely diagnosed $(12,16-18)$. The most common primary site of pPNET is the chest wall, which is known as Askin tumor. The second most common primary site of pPNET is the pelvis $(19,20)$. To the best of our knowledge, among the associated literature, the present case is only the fourth report of laryngeal pPNET (13-15). In addition, to the best of our knowledge, in the 5 years following the presentation of this case, no similar reports have been documented. Previous reported cases of pPNET arising in the larynx are summarized in Table II (13-15).

Histologically, typical pPNET is comprised of small round, blue-stained cells possessing hyperchromatic nuclei, with a high mitotic rate (21). The cytoplasm is indistinct, except in regions where cells are more mature, and the elongated hair-like cytoplasmic extensions coalesce to form rosettes. The majority of the rosettes contain a central solid core of neurofibrillary material known as a lobular or pseudorosette, for example, the Homer-Wright rosette $(13,22)$.

The ultrastructural characteristics of a typical pPNET are primarily the presence of junctional complexes and confluent cell processes, which contain occasional neurosecretory type granules, microtubules and intermediate filaments (23).

Immunohistochemically, a number of authors have reported that typical pPNETs are positive for NSE and S100 protein staining, as well as additional markers, including Leu-7, synaptophysin, neurofilament (24), cytokeratin and chromogranin, which are positive at varying rates, suggesting variation in neural differentiation (25). ES and pPNET apparently arise in association with a translocation between chromosomes 11 and 22, specifically t(11;22)(q24;q12). These two tumors also express glycoprotein $\mathrm{p} 30 / 32$, coded for by the CD99 gene (HBA71 antigen or MIC2), and are recognized by commercially available antibody, O13 (19).

Anti-CD99 antibodies have been recognized as specific markers for the differential diagnosis of ES and pPNET, as these tumors demonstrate strong positive expression of the CD99 gene (26). However, a number of studies have reported that cases of bone and soft tissue tumors, including T-lymphoblastic lymphoma (27), poorly differentiated synovial sarcoma (28), small cell osteosarcoma (29), rhabdomyosarcoma (26), desmoplastic small round cell tumor (30), small cell carcinoma (31), Merkel cell carcinoma (32) and mesenchymal chondrosarcoma (33), also express CD99 gene products (24-31).

Therefore, differential diagnoses of ES and pPNET must be obtained from overall microscopic, ultrastructural and immunohistochemical findings. In addition, identification of a common cytogenetic abnormality $\mathrm{t}(11 ; 22)(\mathrm{q} 24 ; \mathrm{q} 12)$ in these tumors provides strong support for diagnosis.

A number of previous studies have reported that pPNET is an aggressive disease, demonstrating high rates of local recurrence and distant metastases at an early stage. The most frequently observed sites of metastasis are the lungs and bone (34). Numerous patients exhibiting distant metastases at the time of diagnosis of pPNET possessed poor prognoses (35). The treatment for these pPNET cases was similar to that for rhabdomyosarcoma and Ewing's sarcoma; however, a highly effective treatment strategy for pPNETs remains to be established $(36,37)$. In previous years, the prognosis of pPNET was poor, but has improved due to the administration of combined treatment strategies consisting of surgery, radiotherapy and chemotherapy $(34,38)$.

The range of surgical resection is controversial as pPNET arises at a variety of sites, and the method of resection and surgical margin is site-specific. For tumors located in the head and neck, a wide surgical margin cannot be achieved. It has been reported that when pPNETs were resected with a surgical margin $>10 \mathrm{~cm}$, postoperative radiotherapy was not required (34). Regarding the larynx and with consideration of the anatomy in the present study, it was hypothesized that if the tumor did not invade the adjacent tissue, including the pharynx and thyroid, laryngectomy was a wide enough resection of pPNET, as the mucosa of the larynx was enclosed by thyroid and cricoid cartilage. In the present case, the patient refused a laryngectomy due to the fact that the resection was close to the surgical margin and further surgery may have been 
required. A number of previous studies have recommended resection of pPNETs, with a wide surgical margin. It has been reported that patients who underwent a resection with a wide surgical margin, demonstrated improved overall survival, compared with those who underwent a less-than-wide resection (39). Additionally, for patients with localized tumors, wide resection may facilitate favorable prognoses. Additional postoperative radiotherapy may be capable of controlling local disease when microscopic surgical margins remain pathologically positive (34). The resection of all gross pPNET tumors within 3 months of diagnosis is correlated with significantly improved disease-free survival rates (20).

Furthermore, multiagent chemotherapy has been recommended for the control of distant micrometastases by numerous previous studies. Since the 1980 s, preliminary reports have suggested vincristine, ifosfamide, cyclophosphamide and doxorubicin as chemotherapeutic agents for the treatment of metastasis of Ewing's sarcoma. Identical chemotherapeutic strategies have been performed on pPNETs, and the responses were similar to those identified in Ewing's sarcoma, resulting in improved rates of survival $(10,35,38,40)$. However, the optimal chemotherapeutic agents for the treatment of pNET remain under investigation.

In previous studies, the prognosis of pPNET has been demonstrated to be poor, although certain studies have demonstrated improvements $(34,39)$. Current literature indicates that the overall 5-year survival rate is $\sim 40-60 \%$, whereas this figure was $48 \%$ subsequent to 1970 , and $28 \%$ prior to this (34). In recent reports, 5-years survival rate is around 60\% (41-43). However, an improved prognosis has been demonstrated by patients exhibiting pPNET arising in the head and neck region, compared with those patients with tumors at alternative sites $(35,44,45)$. In a previous study, 5 cases of pPNET were reported to have arisen at head and neck sites. In these cases, an improved prognosis was demonstrated, compared with tumors that arose at alternative sites, and all 5 patients had survived 18 months later (46). This result suggested that complete tumor resection with a wide margin, followed by local irradiation and systemic chemotherapy may be concluded to provide an improved prognosis for patients exhibiting pPNET, compared with that of alternative treatment strategies.

In conclusion, pPNET in the head and neck region is rare, particularly in the larynx. pPNET treatment strategies consisting of combinations of surgery, chemotherapy and radiation remain controversial. Further study is required in order to provide clearer evidence regarding the optimal treatment strategy for pPNET.

\section{References}

1. Riggi N and Stamenkovic I: The Biology of Ewing sarcoma. Cancer Lett 254: 1-10, 2007.

2. von Levetzow C, Jiang X, Gwye Y, et al: Modeling initiation of Ewing sarcoma in human neural crest cells. PLoS One 6: e19305, 2011.

3. Batsakis JG, Mackay B and el-Naggar AK: Ewing's sarcoma and peripheral primitive neuroectodermal tumor: An interim report. Ann Otol Rhinol Laryngol 105: 838-843, 1996.

4. Stout AP: A tumor of the ulnar nerve. Proc NY Pathol Soc 12: $2-12,1918$

5. Ewing J: Classics in oncology. Diffuse endothelioma of bone. James Ewing. Proceedings of the New York Pathological Society, 1921. CA Cancer Clin J 22: 95-98, 1972.
6. Angervall L and Enzinger FM: Extraskeletal neoplasm resembling Ewing's sarcoma. Cancer 36: 240-251, 1975.

7. Askin FB, Rosai J, Sibley RK, Dehner LP and McAlister WH: Malignant small cell tumor of the thoracopulmonary region in childhood: A distinctive clinicopathologic entity of uncertain histogenesis. Cancer 43: 2438-2451, 1979.

8. Jaffe R, Santamaria M, Yunis EJ, Tannery NH, Agostini RM Jr, Medina J and Goodman M: The neuroectodermal tumor of bone. Am J Surg Pathol 8: 885-898, 1984.

9. Dehner LP: Peripheral and central primitive neuroectodermal tumors. A nosologic concept seeking a consensus. Arch Pathol Lab Med 110: 997-1005, 1986.

10. Wexler LH, DeLaney TF, Tsokos M, Avila N, Steinberg SM, Weaver-McClure L, Jacobson J, Jarosinski P, Hijazi YM, Balis FM and Horowitz ME: Ifosfamide and etoposide plus vincristine, doxorubicin, and cyclophosphamide for newly diagnosed Ewing's sarcoma family of tumors. Cancer 78: 901-911, 1996.

11. Furman J, Murphy WM, Jelsma PF, Garzotto MG and Marsh RD: Primary primitive neuroectodermal tumor of the kidney. Case report and review of the literature. Am J Clin Pathol 106: 339-344, 1996.

12. Lane S, Ironside JW and Path MRC: Extra-skeletal Ewing's sarcoma of the nasal fossa. J Laryngol Otol 104: 570-573, 1990.

13. Jones JE and McGill T: Peripheral primitive neuroectodermal tumors of the head and neck. Arch Otolaryngol Head Neck Surg 121: 1392-1395, 1995.

14. Yang YS and Hong KH: Extraskeletal Ewing's sarcoma of the larynx. J Laryngol Otol 118: 62-64, 2004.

15. Lynch MC, Baker A, Drabick JJ, Williams N and Goldenberg D: Extraskeletal Ewing's sarcoma arising in the larynx. Head Neck Pathol 8: 225-228, 2014

16. Chowdhury K, Manoukian JJ, Rochon L and Begin LR: Extracranial primitive neuroectodermal tumor of the head and neck. Arch Otolaryngol Head Neck Surg 116: 475-478, 1990.

17. Toda T, Atari E, Sadi AM, Kiyuna M and Kojya S: Primitive neuroectodermal tumor in sinonasal region. Auris Nasus Larynx 26: 83-90, 1999.

18. Ferlito A: Primary Ewing's sarcoma of the maxilla: A clinicopathological study of four cases. J Laryngol Otol 92: 1007-1024, 1978.

19. Weidner N and Tjoe J: Immunohistochemical profile of monoclonal antibody O13: Antibody that recognizes glycoprotein p30/32MIC2 and is useful in diagnosing Ewing's sarcoma and peripheral neuroepithelioma. Am J Surg Pathol 18: 486-494, 1994.

20. Kushner BH, Hajdu SI, Gulati SC, Erlandson RA, Exelby PR and Lieberman PH: Extracranial primitive neuroectodermal tumors. The Memorial Sloan-Kettering Cancer Center experience. Cancer 67: 1825-1829, 1991.

21. Hart MN and Earle KM: Primitive neuroectodermal tumors of the brain in children. Cancer 32: 890-897, 1973.

22. Hachitanda Y, Tsuneyoshi M, Enjoji M, Nakagawara A and Ikeda K: Congenital primitive neuroectodermal tumor with epithelial and glial differentiation. An ultrastructural and immunohistochemical study. Arch Pathol Lab Med 114: 101-105, 1990.

23. Mackay B, Luna MA and Butler JJ: Adult neuroblastoma. Electron microscopic observations in nine cases. Cancer 37: 1334-1351, 1976

24. Yao M, Dornfeld KJ, Buatti JM, Skwarchuk M, Tan H, Nguyen T, Wacha J, Bayouth JE, Funk GF, Smith RB, et al: Intensity-modulated radiation treatment for head-and-neck squamous cell carcinoma - the University of Iowa experience. Int J Radiat Oncol Biol Phys 63: 410-421, 2005.

25. Fellinger EJ, Garin-Chesa P, Glasser DB, Huvos AG and Rettig WJ: Comparison of cell surface antigen HBA71 (p30/32MIC2), neuron-specific enolase, and vimentin in the immunohistochemical analysis of Ewing's sarcoma of bone. Am J Surg Pathol 16: 746-755, 1992.

26. Ambros IM, Ambros PF, Strehl S, Kovar H, Gadner H and Salzer-Kuntschik M: MIC2 is a specific marker for Ewing's sarcoma and peripheral primitive neuroectodermal tumors. Evidence for a common histogenesis of Ewing's sarcoma and peripheral primitive neuroectodermal tumors from MIC2 expression and specific chromosome aberration. Cancer 67: 1886-1893, 1991.

27. Riopel M, Dickman PS, Link MP and Perlman EJ: MIC2 analysis in pediatric lymphomas and leukemias. Hum Pathol 25: 396-399, 1994. 
28. Gerald WL, Ladanyi M, de Alava E, Cuatrecasas M, Kushner BH, LaQuaglia MP and Rosai J: Clinical, pathologic, and molecular spectrum of tumors associated with $\mathrm{t}(11 ; 22)(\mathrm{p} 13 ; \mathrm{q} 12)$ : Desmoplastic small round-cell tumor and its variants. J Clin Oncol 16: 3028-3036, 1998.

29. Devaney K, Vinh TN and Sweet DE: Small cell osteosarcoma of bone: an immunohistochemical study with differential diagnostic considerations. Hum Path 24: 1211-1225, 1993.

30. Scotlandi K, Serra M, Manara MC, Benini S, Sarti M, Maurici D, Lollini PL, Picci P, Bertoni F and Baldini N: Immunostaining of the p30/32MIC2 antigen and molecular detection of EWS rearrangements for the diagnosis of Ewing's sarcoma and peripheral neuroectodermal tumor. Hum Pathol 27: 408-416, 1996.

31. Lumadue JA, Askin FB and Perlman EJ: MIC2 analysis of small cell carcinoma. Am J Clin Pathol 102: 692-694, 1994

32. Perlman EJ, Lumadue JA, Hawkins AL, Cohen K, Colombani P and Griffin CA: Primary cutaneous neuroendocrine tumors. Diagnostic use of cytogenetic and MIC2 analysis. Cancer Genet Cytogenet 82: 30-34, 1995.

33. Granter SR, Renshaw AA, Fletcher CD, Bhan AK and Rosenberg AE: CD99 reactivity in mesenchymal chondrosarcoma. Hum Pathol 27: 1273-1276, 1996.

34. Rud NP, Reiman HM,Pritchard DJ, Frassica FJ and Smithson WA: Extraosseous Ewing's sarcoma. A study of 42 cases. Cancer 64: 1548-1553, 1989.

35. Raney RB, Asmar L, Newton WA Jr, Bagwell C, Breneman JC, Crist W, Gehan EA, Webber B, Wharam M, Wiener ES, et al: Ewing's sarcoma of soft tissues in childhood: A report from the Intergroup Rhabdomyosarcoma Study, 1972 to 1991. J Clin Oncol 15: 574-582, 1997.

36. Harper PG, Pringle J and Souhami RL: Neuroepithelioma - a rare malignant peripheral nerve tumor of primitive origin: Report of two new cases and a review of the literature. Cancer 48: 2282-2287, 1981.

37. Hashimoto H, Enjoji M, Nakajima T, Kiryu H and Daimaru Y: Malignant neuroepithelioma (peripheral neuroblastoma). A clinicopathologic study of 15 cases. Am J Surg Pathol 7: 309-318, 1983.
38. Soule EH, Newton W Jr, Moon TE and Tefft M: Extraskeletal Ewing's sarcoma: A preliminary review of 26 cases encountered in the Intergroup Rhabdomyosarcoma Study. Cancer 42: 259-264, 1978

39. Ahmad R, Mayol BR, Davis M and Rougraff BT: Extraskeletal Ewing's sarcoma. Cancer 85: 725-731, 1999.

40. Kinsella TJ, Triche TJ, Dickman PS, Costa J, Tepper JE and Glaubiger D: Extraskeletal Ewing's sarcoma: Results of combined modality treatment. J Clin Oncol 1: 489-495, 1983.

41. Gupta AA,Pappo A, Saunders N, Hopyan S, Ferguson P, Wunder J O'Sullivan B, Catton C, Greenberg M and Blackstein M: Clinical outcome of children and adults with localized Ewing sarcoma: Impact of chemotherapy dose and timing of local therapy. Cancer 116: 3189-3194, 2010.

42. Rodríguez-Galindo C1, Liu T, Krasin MJ, Wu J, Billups CA, Daw NC, Spunt SL, Rao BN, Santana VM and Navid F: Analysis of prognostic factors in ewing sarcoma family of tumors: Review of St. Jude Children's Research Hospital studies. Cancer 110: 375-384, 2007

43. Smorenburg CH, van Groeningen CJ, Meijer OW, Visser M and Boven E: Ewing's sarcoma and primitive neuroectodermal tumour in adults: single-centre experience in The Netherlands. Neth J Med 65: 132-136, 2007.

44. Hafezi S, Seethala RR, Stelow EB, Mills SE, Leong IT, MacDuff E, Hunt JL, Perez-Ordoñez B and Weinreb I: Ewing's family of tumors of the sinonasal tract and maxillary bone. Head Neck Pathol 5: 8-16, 2011.

45. Kimber C, Michalski A, Spitz L and Pierro A: Primitive neuroectodermal tumours: Anatomic location, extent of surgery, and outcome. J Pediatr Surg 33: 39-41, 1998.

46. Nikitakis NG, Salama AR, O'Malley BW Jr, Ord RA and Papadimitriou JC: Malignant peripheral primitive neuroectodermal tumor-peripheral neuroepithelioma of the head and neck: A clinicopathologic study of five cases and review of the literature. Head Neck 25: 488-498, 2003. 